Bol. Acad. peru. leng. 50. 2010 (191-206)

\title{
CÉSAR VALLEJO EN INDIA
}

\author{
Shyama Prasad Ganguly \\ Universidad Jawaharlal Nehru, Nueva Delhi
}

Fecha de recepción: $\quad$ 03/08/2010

Fecha de aceptación: 30/09/2010

Uno de los estudiosos de la obra de César Vallejo, Jorge Puccinelli, en su valiosísimo trabajo de recopilación de las "Crónicas y Artículos desde Europa" del poeta peruano, comienza su introducción con las siguientes palabras:

“[...] en Vallejo se da la paradoja de ser a la vez el escritor más conocido y en algunos aspectos el menos conocido de la literatura peruana. Vastos sectores de su producción que en apreciable medida pueden contribuir a precisar su imagen, a definir algunos contornos borrosos, a confirmar o rectificar ciertos juicios y a esclarecer en definitiva su obra, continúan dispersos, inéditos, totalmente ignorados."

Aunque dicha situación se haya subsanado en alguna medida en años recientes por el énfasis sobre la revaloración de la obra total de Vallejo, la observación de Puccinelli sigue teniendo validez en general a nivel hispanoamericano o internacional. Y qué duda cabe que es doblemente 
válido ese juicio en el caso de la India en cuanto a la formulación de una idea completa en cuanto a la presencia del poeta peruano en un ámbito tan lejano. Tratar de recopilar la recepción de una figura literaria ajena de antaños en veinticuatro idiomas en que se han desarrollado las literaturas lingüísticas de la India es sumamente difícil, incluso en los casos de figuras más o menos permanentemente registradas en la memoria del público lector por su divulgación mundial tales como Cervantes, Neruda, Lorca, Paz etc. En cuanto a las letras hispanas, desafortunadamente, además se da el caso de que la conservación y disponibilidad fácil de los documentos, que en nuestro caso se refieren a las publicaciones de libros, periódicos o revistas en tantos idiomas, catalogados de una manera centralizada por temas, es todavía un sueño a realizarse en el entorno bibliotecario.

Tratando de trabajar en el tema de nuestra consideración de hoy, en los últimos meses he podido consultar el material disponible principalmente en idiomas como el hindi que es el idioma nacional, el bengalí que es mi idioma materno y un par de idiomas más como el punjabi y malayalam. Espero que este inicio nos pueda llevar a incluir el tema vallejiano en otros idiomas de la India para tener una visión del conjunto en un futuro próximo no muy lejano.

Siendo un comparatista que viene ocupándose de la recepción indo-hispánica en ambos sentidos, el tema de hoy me impulsa a pensar no solo en la presencia vallejiana en la India sino también al revés, es decir, el tema indio en Vallejo. Pues en el poeta, especialmente fijándome en sus crónicas escritas desde Europa, he notado una fuerte intención de un pensar analógico y comparativo cuyo fin por supuesto es resaltar la realidad latinoamericana o peruana. Esto se materializa dentro de una visión total de una sintaxis universal de civilizaciones. Creo que para él, las analogías nacen precisamente porque este no es el otro, lo cual permite establecer paralelos. Aunque las analogías establecen puentes, no pueden suprimir diferencias pero estas diferencias establecen una relación entre dos puentes diferentes. Como ha dicho Octavio Paz tan acertadamente, la analogía es una metáfora en la que la diferencia se hace ilusoriamente una unidad. Por lo tanto, la analogía implica no la unidad del mundo sino su pluralidad, no su naturaleza idéntica sino sus divisiones. Una necesidad interna de ver esta 
pluralidad sin suprimir las particularidades surge de una postura sicológica contra cualquier modelo homogéneo o autoritario, lo cual constituye un hilo conductor de todo su comportamiento social, político y literario.

Aunque la India o los temas indios no son de muy frecuente ocurrencia en los escritos de Vallejo, hemos encontrado algunos datos muy interesantes. Es indudable que el poeta peruano estuviera muy enterado de la figura de Tagore, especialmente después del fracasado viaje de éste al Perú como invitado especial en las celebraciones del centenario de la batalla de Ayacucho en 1924 y la presencia del vate indio en el ámbito latinoamericano a través de la amistad con Victoria Ocampo así como por las traducciones de la pareja Jiménez. En un artículo enviado al periódico El Norte desde París, el 26 de febrero del 1924, donde Vallejo analiza por qué Europa no respeta e ignora a los latinoamericanos, cosa que no puede hacer ni con Japón ni con India, menciona cómo ha bastado un Tagore para que Europa respete y admire a la India "que se anuncia estupenda". Resalta alli, junto con Tagore, la figura de Rubén Darío y Simón Bolívar, cuyas grandezas, piensa, no bastaron para aducir el mismo respeto al continente americano. Sin embargo, en otro lugar piensa que Tagore fue un colaborador de los ingleses sin saber por supuesto la postura de aquel contra éstos, en diversas actuaciones y escritos. En otro reportaje remitido al periódico Mundial el 5 de marzo de 1926, donde se expresa muy preocupado de la lucha de los pueblos basada en las fuerzas centrales del nacionalismo y el internacionalismo, pregunta si en una futura conflagración mundial triunfará la civilización occidental o la oriental. Hace hincapié en el ahínco que ponen los dirigentes, escritores, artistas y profesores de América por dar la mano a sus colegas de Oriente tales como Gandhi y Tagore además de otros líderes de la región islámica, sin ignorar por supuesto la lenta pero profunda influencia del comunismo. En la realidad político-cultural del antagonismo entre los Estados Unidos y Latinoamérica, la percepción de esa posibilidad de acercamiento de América a Asia ocupa el pensamiento de Vallejo en esos años de estancia y actividad intelectual en París. Redunda decir que siempre late en él un firme sentimiento de la latinidad como el centro de la civilización futura. Es curioso notar que, en esa crónica, Vallejo percibe claramente un cariño que empieza a sentir Europa por la America Latina. 
La estancia en París le ayuda en gran medida a reflexionar sobre esa dicotomía civilizacional entre los Estado Unidos y América Latina, que antes hiciera hincapié con tanta profundidad José Enrique Rodó. A menudo vuelve a ese tema, que plantea el conflicto entre el dinamismo materiabutilitario y la gravitación espiritual, que le empuja más hacia la búsqueda del verdadero espíritu humano e integral que debe poseer el hombre. Es quizás esa búsqueda, a raiz de las reflexiones del orientalista francés, Luis Massignon, que por un lado le hace culpar a Occidente de arruinar las filosofías y religiones de Oriente causando desgracia y la pérdida de su alma y que, por otro lado, ahora está siendo reclamado de Europa. A Vallejo, estas afirmaciones de Massignon le convencen e impactan en gran medida. Piensa que brotan de un pensamiento puro sin ser literarias ni diplomáticas, ni son de tipo "de la eclesiásticas cortesanas como las de Tagore". Deducimos de esta referencia que para Vallejo, Tagore servía a los intereses de alguna cancillería "felicitando con voz de pastor a los reyes y dictadores europeos". Es decir, Vallejo busca un tipo de habla que según él sea solamente la de un hombre. Por supuesto, el enfoque principal de la crónica está en que no encuentra ninguna voz como la de Massignon, que habla de la pérdida del alma de otras culturas por causa de Europa.

Es notable la expresión de los desgarros que siente por los fracasos producidos por el encuentro entre America Latina y Europa o Estados Unidos. Incluso para él, el ideal democrático es una forma inapta para la actuación política nacional en las Américas, igual que el ideal comunista, formas ambas en las que caemos víctimas por ser tentadoras y por "nuestra incurable inclinación al plagio fácil y en bruto y nuestra falta del poder asimilativo". Y quizá esto le conduce a acuñar esa expresión tan sencilla pero profunda que es "aprendamos, en primer lugar, a estudiar y comprender y luego a asimilar, lo demás vendrá por si solo" (aparece esta aseveración enVariedades 1023, 8 de octubre 1937).

El constante quehacer que ocupa a Vallejo a nivel cultural para encontrar una salida terapeútica, es decir, su constante esfuerzo de buscar, le lleva al pensamiento de uno de los filósofos indios de gran estima presente en París en los años veinte. La esperanza en una nueva trayectoria de la cultura le hace meditar en la filosofía de J. Krishnamurti. 
al que considera como un nuevo mesías que ya venía con su "Orden de la Estrella" esparciendo un nuevo mensaje espiritual. Vallejo trata de entender por qué se le considera, en la Europa de aquel entonces, una reencarnación de Jesús y aunque en un primer instante simplemente describe desinteresadamente la personalidad y la doctrina de ese "instructor de mundo", pero por su interés en volver al tema un año después, nos hace pensar que llevaba un sincero deseo de adentrarse en el mecanismo de la conducta que aboga la práctica de la perfección de la vida, explayada por Krishnamurti.

Estando plenamente consciente de las divergencias críticas que producen los pensamientos del filósofo indio, parece que Vallejo encuentra en Krishnamurti las huellas de ese hombre integral y espiritual que busca como su ideal. Si observamos las citas que hace de las aseveraciones de Krishnamurti, nos damos cuenta del parecido sentir filosófico que surge del pensar vallejiano como esencia del actuar existencial. Citemos algunos ejemplos, en palabras de Vallejo:

Krishnamurti dice "no aceptéis nada de lo que voy a deciros. No se trata de obedecerme sino de comprenderme. No se trata de una cuestión de autoridad ni de líneas precisas a las que hay que sujetarse ciegamente. Solo se trata de comprendernos y ayudarnos mutuamente. Os hablo de realidades que vosotros podéis percibir directamente, en esta vida o acaso en una vida futura..."

Al hacer alarde de su personalidad le impresiona cómo Krishnamurti empieza negando el principio de autoridad del hombre sobre el hombre. Dice cómo ese mesías indio "ataca a todas las religiones y todos los sistemas filosóficos" no en sus fundamentos dialécticos ni en sus estructuras ideológicas, sino en cuanto ellos se dirigen a invadir y sustituirse al conocimiento directo y a la experiencia personal de cada individuo; luego, citando a Krishnamurti, dice: "el hombre no debe aceptar la enseñanza que del mundo le hacen los demás sino que debe conocerlo todo por sí mismo" y siguiendo esa línea de argumental afirma Vallejo que para el nuevo profeta de la India "todos los filósofos, artistas, héroes y sacerdotes que han evangelizado tal y cual disciplina de la vida no han hecho más que interponer 
entre la verdad y los ojos de cada uno de los demás individuos, la pantalla de un tercero, es decir, de un intruso". Para él, Krishnamurti es un apóstol de suprema liberación que quiere combatir toda credibilidad y enseñar a los hombres a encontrar por sí mismos la verdad “que está en nuestro propio corazón y en nuestra experiencia personal". Es en este enfoque donde se luce un sendero verdadero para el hombre. Vallejo encuentra de extrema significación los cuatro imperativos categóricos de Krishnamurti que son: el discernimiento, el desinterés, la buena conducta y el amor.

Ese poeta de Hispanoamérica al que hoy conocemos como el más audaz precursor de un nuevo camino literario y cultural, encontró en Krishnamurti un hermano asiático que pregonaba la evolución del hombre hacia la liberación destruyendo el principio de autoridad y construyendo la fuerza evolvente y objetiva de la cultura circulante.

Al hombre de tan extrema sensibilidad como Vallejo, la hermandad espiritual de este tipo inscribe su presencia duradera de manera silenciosa.

Indagando en la presencia de la produción y del espíritu político- literario de Vallejo en la India, prestemos atención ahora al trato que ha recibido el poeta peruano en nuestro entorno. A mi modo de ver, son dos los puntos fundamentales que emanan del Corpus literario mismo de César Vallejo. El primero es la presencia del dolor como concepto que brota de la experiencia personal y se convierte en la expresión poética y social a nivel colectivo. El segundo es la expresión de la inconformidad con toda forma de opresión autoritaria. Se me ocurre lamentar que a pesar del conocimiento de la estatura de Tagore, Vallejo no tuviera la oportunidad de adentrarse en la poesía de aquel representante supremo de la poesía del Oriente, a quien simplemente no dio trato literario al juzgarle como generador de una literatura 'eclesiástica cortesana'. Toda la poesía de Tagore, y especialmente sus canciones, se arraigaban en un profundo sentimiento de dolor y sufrimiento al que convirtió en la expresión poética sublime. En sus últimos años, Tagore dejó grabado este 
sufrimiento en los siguientes versos: "En las palabras de sangre/yo vi / mi ser. /Yo me conocí/median te injurias/y dolor. /La verdad es dura/y nunca engaña. /Amé a esa dureza". Teniendo el privilegio de poder asomarme a la poesía de ambos poetas como lector de ambas culturas, veo abundantemente reflejada la aseveración que hiciera Antenor Orrego acerca de la fuente desde donde brota y crece verdaderamente toda poesía cuando dijo que "el dolor es camino de comprensión y de revelación o sino no tendría sentido. Solo así se llega a cierta serenidad". Pensando en términos de Vallejo, sólo los grandes poetas pueden encontrar la palabra y el acento más justo para expresar ese sentimiento. El dolor se convierte en canción humana tanto en Vallejo como en Tagore. Uno no sabe porqué no ocurrió el encuentro entre los dos, ni siquiera literario. Para nosotros fue la pérdida de una de esas grandes oportunidades de contacto literario entre los dos espacios. Podemos conjeturar también que en alguna pequeña medida el desinterés de Vallejo en la India hubiera podido surgir de no poder conocer y apreciar la obra y la personalidad del vate indio. Cabe mencionar que no podemos llevar esta aseveración a sus últimas consecuencias. El hecho de que no haya habido la merecida presencia vallejiana entre el público lector indio, ni entre los artistas y movimientos artísticos progresistas, a pesar de la amplia circulación de nombres como Neruda y Lorca, es un tema curioso. No cabe duda de que el martirio de Lorca y la abundante circulación física, los trastornos políticos que enfrentó y sobre todo el Premio Nobel de Neruda, les consagraron como los poetas vitales más representivos del ámbito hispano en un país que se veía sumergido en una lucha antiimperialista, con tantos problemas opresivos sociales.

Pero a pesar de ser injustamente ignorado al lado de esas otras presencias, durante las últimas décadas ha habido una creciente consciencia de la importancia de Vallejo en los distintos idiomas de la India. Esto se nota por la referencia a su personalidad y obra por poetas e intelectuales, así como por las traducciones. No lo podemos comparar con el crecimiento firme de la gloria de Vallejo en el ámbito hispano u occidental como fuerza viviente y fecunda, sino simplemente como un deseo de reconocerle como "una energía de la historia, una influencia viviente de un hombre sobre los demás" a través de las traducciones de literatura extranjera en nuestro 
país. Queremos subrayar ahora sólo algunos ejemplos de esta presencia puesto que, como hemos señalado anteriormente, es una tarea gigantesca recopilar todo lo que haya salido sobre el poeta en los 24 idiomas del país. Hay que añadir a esas desconocidas cifras el gran número de lectores que se acceden a la literatura extranjera via el idioma inglés.

Entre esta plétora de idiomas, me voy a basar en la recepción selectiva en tres idiomas que son hindi, bengali y punjabi/gurumukhi. Por supuesto que no pretendo presentar esta recepción de manera cronológica. Muchas de las revistas literarias en los distintos idiomas han publicado algunas que otras traducciones de la poesía de Vallejo aunque éste no hubiera sido tema importante del discurso literario de muchos poetas de la manera en que lo fueron Lorca o Neruda. En hindi dos colecciones antológicas en forma de homenaje muestran la predilección y el gusto de los traductores, académicos y los lectores en cuanto a la selección de material vallejiano. La primera fue realizada en los años sesenta por la conocida traductora Premiata Verma que en su libro publicado en 1969 presenta una selección principalmente de los poemarios Los heraldos negros y Poemas humanos con la inclusión de alguno que otro poema de Trilce y de España aparta de mi este Cáliz. Citemos los títulos de algunas selecciones de "Los heraldos negros": por ejemplo, "El poeta a su amada", "Idilio muerto", "Líneas", “Capitulación”, "La cena miserable”, "El tálamo eterno”, "Los dados eternos", "Los anillos fatigados", "Dios", “A mi hermano Miguel”, "Los pasos lejanos", "Esperjesias" y poemas como "La rueda de hambriento", "Hoy me gusta la vida mucho menos", "Piedra negra sobre una piedra blanca", "Acabo de pasar el que vendrá" (de la colección Poemas humanos) $e$ incluso de algunos de Trilce como el poema que empieza con el verso "Esta noche desciendo del caballo". La otra colección antològica salió como resultado de un seminario sobre Vallejo organizado por la Universidad de Delhi en 1992 que, asimismo, incluye una representación de poemas de las colecciones mencionadas anteriormente pero también un ejemplo de los poemas en prosa. Esta colección de traducciones al hindi incluye trabajos de Premiata Verma, Rachna Chauhan, Tanuka Chakravorty. Como fue producto de un seminario, esta colección incluye ensayos en inglés sobre el corpus poético de Vallejo por intelectuales e hispanistas de la India y España reflexionando sobre diversos aspectos de los poemarios 
Los heraldos negros y los Poemas humanos así como una aportación sobre la reflexión de la Guerra civil española en César Vallejo.

Es curioso que en las traducciones realizadas en los distintos idiomas, se han visto pocos ejemplos de los poemas de Trilce, de Los poemas en prosa e incluso de creaciones tan importantes como "Himno a los voluntarios de la república" y del conjunto de poemas de España aparta de mi este cáliz. O cuando mucho su aparición es tardía y esto es doblemente válido en el caso de Trilce. Esto evidencia, por un lado, el deseo de los poetas de captar a aquel Vallejo que versifica sobre la condición humana, aplicable a todas las sociedades donde prevalecen condiciones sociales de injusticia y opresión. Asimismo, se hace más manejable los problemas de las equivalencias interculturales y no resulta nada problemático domesticar a una literatura extranjera. Pero creo que el verdadero reto de adquirir una comprensión cabal de Vallejo en nuestro entorno estribaría en lograr que se haga una antología crítica detallada en algún idioma indio de su poemario Trilce. Los pocos poemas de esta colección que existen en las versiones indias resultan bastante inadecuadas, ya que o son los más sencillos o inadecuados por no proporcionar las aclaraciones necesarias a varios neologismos, arcaísmos o modificaciones lexicales que encierran bastante hermetismo y donde la simbolización es extremadamente personal o de una autoctonía cultural en donde quedan escondidos los posibles significados de los poemas. En cuanto a la calidad de otros poemas, la mayoría son bien logradas y fácilmente apelan al lector público sin causar ninguna enajenación. Para no extenderme, evito citar ejemplos concretos de traducción pero creo que en un trabajo más amplio valdría la pena disertar sobre ese aspecto.

Otra publicación importante de tema vallejiano en la India fue el número especial de Hispanic Horizon, única revista hispánica que sale de la India, publicada por el Centro de Estudios Hispánicos de la Universidad Jawaharlal Nehru. En su número 6 y 7 de 1988-89 se incluyen diferentes aportaciones sobre el significado de César Vallejo para las letras contemporáneas internacionales haciendo referencia a algunas de sus obras. Algunas de estas aportaciones fueron presentadas en un seminario sobre el poeta organizado por el mismo Centro. La portada del número 
reproduce la imagen gráfica de una obra teatral de Vallejo (La piedra cansada), aspecto que ha sido casi totalmente ignorado en la India. Cabría pensar en sacar alguna versión india de uno de los bocetos originales de los que me informa recientemente Ricardo González Vigíl, experto peruano en la obra del maestro (tales como "Suite y contrapunto", "Dressing Room" o "Charlot contra Chaplin" además de las obras conocidas y ya sacadas anteriormente) y publicarlo como proyecto especial de dicha revista de la JNU o de algún seminario de la Universidad de Delhi.

Lo subrayo ya que este Centro y el referido departamento de la Universidad de Delhi son las únicas instituciones que programan la consideración académica de Vallejo en los cursos universitarios de manera continua.

En uno de los artículos incluidos en el referido número de Hispanic Horizon, el hispanista indio, el Prof. S. Dey, reflexionando de manera comparativa sobre los aspectos vitales en la creación de Vallejo, hace referencia a la importancia del tema del pan y hambre como recuerdo de la infancia y comenta sobre la coincidencia de que este mismo aspecto se nota en el poeta bengalí Sukanto Bhattacharya, poeta con semejantes recuerdos de hambre y dolor, quien compara a la luna con un roti quemado (el roti es la forma del pan redondo indio). Esta comparación insinúa lo que va a ser un aspecto común entre Vallejo y muchos poetas indios que se centran en la expresión de la condición humana dolorosa como el predicamento principal de sus obras poéticas.

En la lengua bengalí, como hemos dicho antes, la presencia de Lorca y Neruda se registran muy temprano durante los años cuarenta. Pero César Vallejo es objeto de alguna atención por parte de magazines y revistas literarias mucho después, es decir, no antes del comienzo de la década de los sesenta. No ha sido posible recopilar todos esos datos pero la presencia más palpable de nuestro poeta se registra en el trabajo del traductor y comparatista renombrado de Bengala, el Prof. Manabendra Bandopadhyay. El venía traduciendo poemas de Vallejo desde los sesenta. Algunos de estos han sido presentados en su antología en la lengua bengalí la cual se titula $E l$ sueño, el destino: los mejores poemas rebeldes 
latinoamericanos. La selección aquí también es muy representativa, tan sólo incluye cuatro poemas seleccionados de Los poemas humanos ("Los nuevos monstruos", "Traspié entre dos estrellas" y "Los degraciados") y de la colección "Poemas en prosa" ("Voy a hablar de la esperanza"). Pero la gran aportación del trabajo es la ubicación de Vallejo en el contexto de la evolución de la poesía de protesta ante las condiciones de opresión y discriminación en el proceso socio-cultural de América Latina. Se encuentra allí toda una pléyade de poetas que representan la tradición de "voces rebeldes". En el ambiente progresista de la literatura bengalí, que poco a poco se agudiza con el movimiento progresista de los intelectuales en todo el país en torno al fenómeno de la independencia y el impacto tanto del pensamiento socialista como de las otras corrientes influyentes de la modernidad, era natural que temprano o tarde figuras como Vallejo aparecieran progresivamente en el escenario literario de la india. El mismo especialista indio Bandopadhyay ha sacado otro libro en bengalí acerca de la poesía antifascista intitulado La guerra Civil española, cincuenta años después, en que presenta quince piezas de Vallejo sobre ese tema de España aparta de mí este cáliz. Son libros muy divulgados entre los poetas, intelectuales y los sectores universitarios de Bengala Occidental y Bangladesh. Otro académico y traductor bengalí, Rabin Pal, escribiendo en una célebre revista Proma en su número de julioseptiembre de 1995, introduce una novedad que consiste en incluir, entre los 14 que traduce, seis poemas de Trilce (Poemas XXXIII, XV, XVI, XLV, XIV, XLIV), otros 4 de España, aparta de mí este cáliz, 2 de Poemas humanos ("La cólera que quiebra..." y "Los desgraciados"), y 2 de Los heraldos negros ("La araña” y "El pan nuestro") además de una reflexión bien contemplada del significado del poeta como una de las más puras y vitales expresiones de la condición humana. El énfasis sobre Trilce es un signo positivo por razones obvias. Para mi sorpresa he encontrado datos de otro texto de Vallejo en bengalí (Jiban-Mrityur Opare), traducción de "Más alla de la vida y la muerte" realizada por Amitabha Sarkar y publicada en la colección de cuentos Shei Mohonaar Dhaare editada por Debesh Ray. El hecho de que todas estas traducciones en bengalí se hayan basado en versiones ingleses provenientes del extranjero responde a algo inevitable y de larga tradición. Tanto es el ahínco que ponen los trauctores bengalíes en la primacía o relevancia de la versión traducida que no piensan necesario mencionar 
ni siquiera los títulos originales en español ni sus equivalentes en inglés. Esta situación en cuanto a las traducciones indirectas no va a cambiar hasta que se genere un suficiente número de traductores capacitados en el ámbito del limitado hispanismo en la India. El debate indio sobre el valor de las traducciones ante el texto original es muy candente pero no nos cabe entrar en ese tema aquí.

En mi búsqueda del material relevante a este trabajo también me tropecé con la traducción de cuatro poemas en Gurumukhi, lengua del Punjab que está al norte de la india. Estas traducciones han salido en 2005 en la revista Hunn (el momento actual) realizadas por el poeta Avtar Jandyalvi. Uno de los poemas es "Masa" de España aparta de mi este cáliz y otros son de Los heraldos negros.

Una de las consecuencias de la distancia en el tiempo y espacio y los impactos de los procesos históricos y sociales acuciantes es que se tomen prestado de esas personalidades los ejemplares del extranjero que tienen más relevancia actual. Por eso, del entorno total de su obra privilegia ese enfoque de su poesía en que se evidencia claramente su compromiso con la sociedad y el ser humano. En la actualidad han aparecido publicaciones que resaltan las tendencias principales de la poesía latinoamericana en el siglo XX. Además del libro ya mencionado de Manabendra Bandopadhyay, recientemente la Academia de Letras de India, en colaboración con el GRULAC (una Asociación que representa el grupo de los países latinoamericanos y caribeños), ha publicado una antología poética importante de dicha región, donde se aclara en qué medida Vallejo representa el comienzo de la modernidad en la poesía latinoamericana. La aparición del número especial de la revista, arriba mencionada, también es prueba de un interés vivo en Vallejo entre los sectores universitarios. Pero todavía constituye una "disjecta membra" de un corpus más amplio que algún día se tendrá que rastrear y rescatar para ofrecer una visión del conjunto que reclama el poeta.

Del tipo de interés que genera Vallejo entre los medios intelectuales y lectores de poesía me hace pensar que su importancia estriba en ser uno de los representantes de esa 'unidad excelsa', de esa totalidad que no se suma 
sin integrarle a él como parte de ese conjunto en que figuran también otros poetas como Neruda y Guillen el conjunto que introduce una nueva visión de poesía y los que fueron responsables para establecer las pautas para el camino poético del futuro. Para los lectores de poesía en la India estos tres poetas representan las tres fuerzas más potentes que inician la poesía rebelde en Hispanoamérica, en el sentido de ser los iniciadores de una poesía que asentó firmemente la posibilidad de una expresión poética que pudo conjugar idealmente la doble responsabilidad reclamada por el compromiso social y la libertad artística. Para citar de los escritos de Vallejo mismo, esa expresión poética detenía "el agotamiento del contenido social de las palabras" y no permite "interrumpir la relación articulada del hombre con otros hombres" (Univ. U.M.S.M. No.2, Lima, 1 de oct., 1931)

Históricamente, el tránsito desde la época colonial a la descolonización en la India resalta la primacía de las consideraciones populares. Los movimientos sociales y políticos también tienden a introducir los cambios necesarios para poner al hombre común en el primer plano. Dentro del país los movimientos literarios también registraban y promovían estos cambios. Entonces no era nada sorprendente que la introducción de poetas como Neruda y Lorca, no sólo en cuanto a su aportación poética sino también en cuanto a su envergadura de hombres inconformes con el estado de las cosas, encontrara un camino ya asfaltado en la India. Cambiaba no sólo la situación social y política sino también la expresión y el pensamiento mismo. En la búsqueda de esa expresión auténtica iban a ser ejemplos ideales esos poetas, incluyendo a Vallejo, quienes construyeron esa identidad propia de su entorno abogando una revolución no sólo en el pensamiento sino en el lenguaje. Explica Manabendra Bandopadhyay muy acertadamente que fueron esos poetas que, según Roberto Fernández Retamar, presentan a Calibán como el símbolo del hombre hispanoamericano ante todo tipo de opresión al que fue sometido. Estos poetas entonces rescatan a Calibán de su simbolización anterior que Rodó se lo quiso dar hace algunas décadas anteriores. La India poscolonial, ciertamente, representaba ese estado de evolución revolucionaria que ocurrió en América Latina en su tránsito desde el Calibán de José Enrique Rodó al Calibán de Retamar donde la gente común vuelve al primer plano. En su mismo seno los poetas de India necesitaban y llevaban a cabo muchos experimentos audaces para 
inscribir esa transformación. Cómo no iban de repercutir en su conciencia la aportación de los tres grandes de América Latina que anuncian la nueva época. En las nuevas coyunturas de siglo XX, los poetas de distintos países llevaban el mismo sueño y por medio de ellos hablaron los pueblos de los continentes, pueblos a los que convenía una poesía más sencilla de elementos cotidianos: el pan, el sudor, el trabajo diario, el amor etc. etc. La tarea creativa era de poetizar esa experiencia común y corriente con un espíritu libre sin dogma según el cual la experiencia era la que debía formular la acción teórica y no al revés. Este último precepto orientó a Vallejo toda la vida. Es una visión que figura como concepto central del arte para los artistas como Vallejo que no quieren aceptar ninguna consigna o propósito que someta su libertad estética al servicio de alguna propaganda política.

En su introducción a la recopilación de las aportaciones al seminario en torno a Vallejo en la universidad de Delhi antes referido, el entonces embajador del Perú, el finado Sr. D. Alejandro San Martin, hombre de excelente preparación literaria, se preguntaba cómo hablar de Vallejo en Delhi. Para él la relectura de Vallejo en la India fue una rara experiencia pero encontró en el contexto peruano e indio, a la vez distante e inmediato, muchas similitudes muy vivas. Pero al mismo tiempo ese diplomático insinúa la perfecta armonía de esa personalidad y de sus experiencias en nuestro contexto. Indagando en esa impresión, me dirigí en otra ocasión a otro excelente literato peruano que actualmente sirve de alto diplomático en la embajada peruana en la India. En una charla presentada en nuestra universidad hace tan sólo algunos meses sobre Neruda y Vallejo, este diplomático, el Sr. Carlos Alberto Yrigoyen, me reveló cómo en la realidad inmediata tan acuciante de la India, las palabras de Vallejo cobran y recobran vitalidad. Sus significados se levantan de nuevo, las metáforas se hacen más aplicables y todo el que haya leído a Vallejo observa en qué medida su poesía se magnifica en este tejido social que es la India y el peso de la esperanza sobre un nuevo valor de la solidaridad se rebela como más patente y deseado. Pienso que sólo de esa manera el microcósmico Santiago de Chuco adquiere una dimensión macrocósmica, de la misma manera que García Márquez convirtiera a Macondo en un lugar universal. Pienso que en la India actual Vallejo se 
ha vuelto más relevante para todos aquellos creadores que como él buscan la escencia de la palabra para adecuarse a la realidad verdadera. Con todas estas voces se hermana Vallejo, pues ya atravesamos tiempos en que no sólo basta buscar la palabra exacta para nombrar las cosas como deseaban nuestros más exquisitos creadores de la estética tagoriana. Necesitamos de voces que no sólo nos ayuden a descifrar el significado del mundo a través de la palabra poética exacta sino de las voces que busquen "la palabra más justa y el acento más justo para mover el mundo", como él mismo decía con Joseph Contad. Vallejo es de suma relevancia en la India de hoy.

Para concluir, les quiero informar que en el futuro inmediato tenemos programada la publicación de una antología de Vallejo en hindi traducida directamente del español, la cual va a incluir muestras de la creatividad de Vallejo en todos los géneros. La selección de los textos la hemos realizado a través de un equipo de especialistas y poetas que incluían al finado poeta peruano Javier Sologuren y Germán Belli, entre otros. Además, la embajada peruana en la India proyecta sacar una antología de la traducción de la poesía de Vallejo que incluya por lo menos un texto del poeta en cada una de las veinticuatro lenguas de la India.

\section{BIBLIOGRAFÍA}

VALLEJO, César. Desde Europa, Crónicas y Artículos (19234938), Jorge Puccinelli, 1987, Lima.

BANDOPADHYAY, Manabendra. Eí swapna ei Gantabya, Latín Americar Shreshtha Vidrohi Kavita, Dey's Publishing, 1987, Calcutta.

BANDOPADHYAY, Manebendra(ed). Spener Grihayudha. Panchash Bachor pore, Dey's Publishing, 1989, Calcutta.

PAL, Rabin. “César Vallejo’ Kavi o Kavita” Proma, July-Sep, 1995, Calcutta. 
SARKAR, Amitabh. (Jeevan-Mrituya opare) Traducción del cuento "Mas allá de la vida y la muerte", Protikhyay, Devesh Roy, 1995, Calcutta.

MAURYA, Vibha (ed). Cesar Vallejo- A Homage, Embassy of Peru and University of Delhi, 1992, Delhi.

VERMA, Premiata. César Vallejo Ki Kavitaayen, 1969, Delhi.

AVTAR, Jandyalvi. César Vallejo, *(Poemas en Punjabi), Huun, jun- nov, 2005.

GANGULY, S.P y SUNDRIYAL, Minakshi. Esta Riqueza Abandonada, Sahitya Akademi y GRULAC, 2006, New Delhi.

DEY, S. "Reflection of Life in the Letters of César Vallejo", Hispanic Horizon, no. 6 and 7, (focus on César Vallejo), Centre of Hispanic Studies, JNU, 1988-89, New Delhi.

VALLEJO, César. Obra Poetica Completa, Mosca Azul Editores, 1983, Lima.

Correspondencia:

Shyama Prasad Ganguly

Universidad Jawaharlal Nehru, Nueva Delhi. 